\title{
Order one invariants of immersions of surfaces into 3-space
}

\section{Tahl Nowik}

Received: 3 January 2002 / Revised version: 6 June 2002 /

Published online: 13 November 2003 - (C) Springer-Verlag 2003

\begin{abstract}
We classify all order one invariants of immersions of a closed orientable surface $F$ into $\mathbb{R}^{3}$, with values in an arbitrary Abelian group $\mathbb{G}$. We show that for any $F$ and $\mathbb{G}$ and any regular homotopy class $\mathcal{A}$ of immersions of $F$ into $\mathbb{R}^{3}$, the group of all order one invariants on $\mathcal{A}$ is isomorphic to $\mathbb{G}^{\aleph_{0}} \oplus \mathbb{B} \oplus \mathbb{B}$ where $\mathbb{G}^{\aleph_{0}}$ is the group of all functions from a set of cardinality $\aleph_{0}$ into $\mathbb{G}$ and $\mathbb{B}=\{x \in \mathbb{G}: 2 x=0\}$.

Our work includes foundations for the study of finite order invariants of immersions of a closed orientable surface into $\mathbb{R}^{3}$, analogous to chord diagrams and the 1-term and 4-term relations of knot theory.
\end{abstract}

Mathamatics Subject Classification (2000): 57M, 57R42

\section{Introduction}

The notion of finite order invariants arose in knot theory. It is extended here to the setting of stable immersions of a closed orientable surface into $\mathbb{R}^{3}$. We give a general analysis of finite order invariants, finally classifying all order one invariants (Theorem 5.1). A small subclass of order one invariants has previously been studied, namely, the "local invariants", studied in [MB], [G], [N1], [N3].

The structure of the paper is as follows: In Section 2 we describe the selfintersection appearing in immersions which correspond to the codimension 1 (abbreviated codim 1) strata in the space of immersions; they will be the basis for our definition of finite order invariants. In Section 3 finite order invariants are defined, without referring to a globally defined co-orientation for the strata in the space of immersions; indeed it is shown that such continuous choice of co-orientation does not exist. We construct the combinatorial object on which order $n$ invariants are defined up to order $n-1$ invariants. We then obtain a first relation satisfied by invariants of order $n$, which is related to the non-existence of co-orientations. In Section 4 we obtain the relations on invariants arising from local 2-parameter families of immersions. In Section 5 we classify all order one invariants by analyzing essential loops in the space of immersions. In Section

T. NOWIK

Department of Mathematics, Bar-Ilan University, Ramat-Gan 52900, Israel

(e-mail: tahl@math.biu.ac.il)

Partially supported by the Minerva Foundation 
6 we use our classification of order one invariants to produce an infinite list of equations which are satisfied by any eversion of the sphere. This extends a list of four such equations appearing in [G]. In Section 7 it is shown that for invariants of order $n>1$ with values in $\mathbb{G}=\mathbb{Z} / 2$, the relations we have obtained are insufficient. In that the analysis of [N2] is also completed. Finally it is shown that for any Abelian group $\mathbb{G}$ there exist invariants of any order $n$ with values in $\mathbb{G}$. The problem of classifying all invariants of order $n>1$ remains open.

\section{Self intersection}

Let $F$ be a closed orientable surface and denote by $\operatorname{Imm}\left(F, \mathbb{R}^{3}\right)$ the space of all immersions of $F$ into $\mathbb{R}^{3}$, with the $C^{1}$ topology. The strongest equivalence between immersions $i: F \rightarrow \mathbb{R}^{3}$ that we consider is the following:

Definition 2.1. A regular homotopy $H_{t}: F \rightarrow \mathbb{R}^{3}$ will be called of type A if it is of the form $H_{t}=U_{t} \circ i \circ V_{t}$ where $i: F \rightarrow \mathbb{R}^{3}$ is an immersion and $U_{t}: \mathbb{R}^{3} \rightarrow \mathbb{R}^{3}, V_{t}: F \rightarrow F$ are isotopies. Two immersions $i, j: F \rightarrow \mathbb{R}^{3}$ will be called A-equivalent if there is a regular homotopy of type $A$ between them.

The division of $\operatorname{Imm}\left(F, \mathbb{R}^{3}\right)$ into A-equivalence classes, forms a stratification of this space. An immersion $i: F \rightarrow \mathbb{R}^{3}$ is called stable if it is an interior point of its A-equivalence class. This clearly holds iff the class itself is open, and those are the strata of codim 0 . The codimension of the stratum to which a given immersion belongs depends on the self intersection appearing in that immersion, see [HK] for local analysis. Parts of our work will also be local: given an immersion $i: F \rightarrow \mathbb{R}^{3}$ and a self intersection point of $i$, we will look at all deformations of $i$ which move $F$ only in a small neighborhood of that intersection point. The local stratification will then mean the stratification of this smaller space of immersions and local codimension of the local strata will again refer to this smaller space. We will take a close look at the local stratification up to codim 2 . The codim 0 strata are, as mentioned, the A-equivalence classes of stable immersions. Their self intersection is everywhere in general position i.e. locally equivalent to the intersection of two or three coordinate planes in $\mathbb{R}^{3}$. In this section we will discuss the codim 1 strata. The codim 2 strata will be discussed in Section 4.

The codim 1 strata are divided into four types which (following [G]) we call: $E, H, T, Q$. In the notation of $[\mathrm{HK}]$ they are respectively $A_{0}^{2}\left|A_{1}^{+}, A_{0}^{2}\right| A_{1}^{-}, A_{0}^{3} \mid A_{1}$, $A_{0}^{4}$. The four types may be demonstrated by the following local representatives, where formulae in 3-space defining the different sheets involved in the self intersection, are given. Representatives of the codim 1 strata are obtained from the formulae below by setting $\lambda=0$. Letting $\lambda$ vary, we obtain a 1-parameter family of immersions which is transverse to the given codim 1 stratum.

$E: \quad z=0, \quad z=x^{2}+y^{2}+\lambda$. See Figure 3, ignoring the vertical plane.

$H: z=0, z=x^{2}-y^{2}+\lambda$. See Figure 4, ignoring the vertical plane. 
$T: z=0, \quad y=0, \quad z=y+x^{2}+\lambda$. See Figure 5, ignoring the vertical plane $x=0$.

$Q: \quad z=0, \quad y=0, \quad x=0, \quad z=x+y+\lambda$. This is simply four planes passing through one point, any three of which are in general position.

A self intersection of local codim 1 (namely $E, H, T$ or $Q$ ) will be called a $\mathrm{CE}$ point (after the first and last letters of "co-dimension one"). By CE point we will also refer to the point in $\mathbb{R}^{3}$ where this self-intersection takes place. Once an orientation is chosen for the surface, the above four types of self intersection split into twelve types, as we explain below. A choice of one of the two sides of the local codim 1 stratum at a given point of the stratum, is represented by the choice of $\lambda<0$ or $\lambda>0$ in the formulae above. We will refer to such a choice as a co-orientation for the configuration of the self intersection. A completely different notion of co-orientation that we will encounter is the following: If $i: F \rightarrow \mathbb{R}^{3}$ is an immersion and $F$ is oriented then its orientation and that of $\mathbb{R}^{3}$ induce a co-orientation for $i(F)$ in $\mathbb{R}^{3}$. To avoid confusion between the two notions, we will use the term "co-orientation" only for the former. For the latter we will speak of the "preferred side of $i(U)$ in $\mathbb{R}^{3}$ " wherever $U \subseteq F$ is embedded by $i$.

We now present the twelve types of self intersection in the oriented setting and specify a co-orientation wherever possible:

Types $E$ and $T$ : the configuration of the self intersection at the two sides of the stratum is distinct even with no orientation on the surface, namely, for $\lambda<0$ there is an additional 2-sphere in the image of the immersion, and we choose this side $(\lambda<0)$ as our positive side for the co-orientation. Now, for type $E$ (respectively type $T$ ) this additional 2 -sphere is made of two 2-cells (respectively three 2-cells). If the surface is oriented then we may distinguish three (respectively four) different types of $E$ (respectively $T$ ) self intersections, which we denote by $E^{a}$ (respectively $T^{a}$ ) where $0 \leq a \leq 2$ (respectively $0 \leq a \leq 3$ ) denotes the number of such 2-cells for which the little 3-cell bounded by the 2-sphere lies on their non-preferred side in $\mathbb{R}^{3}$.

Types $H$ and $Q$ : The configuration of the self intersection on the two sides of these strata are indistinguishable without an orientation on the surface. For oriented surfaces we proceed as follows: Let $V$ denote the region between the sheets of the surface which appears once we move away from the stratum (i.e. once $\lambda \neq 0$ ). For type $Q$ this is a simplex defined by our four sheets. For type $H$ this region is not bounded by the local configuration, but may still be defined, e.g. for $\lambda>0$ in the formula for $H, V$ will be a region consisting of points close to the origin and satisfying $0 \leq z \leq x^{2}-y^{2}+\lambda$.

We may now count the number $a$ of sheets for which $V$ is on their preferred (or non-preferred) side. On the other side of the stratum this number will be $2-a$ for type $H$ and $4-a$ for type $Q$. The un-ordered pair of numbers $\{a, 2-a\}$ serves to split the $H$ configuration into two types $\{2,0\}$ and $\{1,1\}$ which we name respectively $H^{2}$ and $H^{1}$. Similarly the un-ordered pair $\{a, 4-a\}$ splits $Q$ into three 
types $\{4,0\},\{3,1\},\{2,2\}$ which we name respectively $Q^{4}, Q^{3}, Q^{2}$. Furthermore, in case this is a pair of distinct numbers, i.e. for $H^{2}, Q^{4}, Q^{3}$, then it may be used to define a co-orientation for that configuration. For $H^{2}$ we choose as the positive side of the stratum, that side where both sheets have $V$ on their preferred side. For $Q^{a}, a=4,3$, we choose as the positive side of the stratum, that where $a$ sheets have $V$ on their non-preferred side. Note that our choice for $H$ and $Q$ uses an opposite convention. Our convention for $Q$ resembles that which we used for $E$ and $T$. The reason we made an opposite choice for $H$ is only to avoid some minus signs in the sequel.

Finally, the two sides of the $H^{1}$ and $Q^{2}$ strata are indistinguishable even when the surface is oriented. This fact is expressed in the following proposition:

Proposition 2.2. Let $B$ be a ball neighborhood in $\mathbb{R}^{3}$ of a CE of type $H^{1}$ or $Q^{2}$. There exists an orientation preserving diffeomorphism $h: B \rightarrow B$ which maps the configuration onto itself (permuting the sheets), being orientation preserving on each sheet, but reversing the co-orientation of the configuration (i.e. motion of the configuration into one side of the stratum corresponds under $h$ to motion into the other side).

We will show that this local symmetry of the $H^{1}$ and $Q^{2}$ configurations implies a global one-sidedness of the $H^{1}$ and $Q^{2}$ strata in $\operatorname{Imm}\left(F, \mathbb{R}^{3}\right)$, i.e. there is no global continuous choice of co-orientation for the $H^{1}$ and $Q^{2}$ strata, (see Remark 3.7 below).

We have seen that for an oriented surface there are twelve different types of CEs. We have attached a symbol to each type and have chosen a co-orientation for all but two of them. This set of twelve symbols will be denoted $\mathcal{C}$. For the sake of convenience we extend $\mathcal{C}$ to a set $\widetilde{\mathcal{C}}$ of fifteen symbols, adjoining the symbols $H^{0}, Q^{0}, Q^{1}$. The new symbol $H^{a}$ (respectively $Q^{a}$ ) will denote the same configuration as $H^{2-a}$ (respectively $Q^{4-a}$ ) only with opposite co-orientation. The co-orientation attached to the new symbols is consistent with that attached to the old ones, namely for any $H^{a}, a \neq 1$ (respectively $Q^{a}, a \neq 2$ ) the positive side of the stratum is where $a$ sheets have $V$ on their preferred side (respectively non-preferred side).

\section{Finite order invariants}

We fix once and for all a closed oriented surface $F$ and a regular homotopy class $\mathcal{A}$ of immersions of $F$ into $\mathbb{R}^{3}$. We denote by $I_{n} \subseteq \mathcal{A}(n \geq 0)$ the space of all immersions in $\mathcal{A}$ which have precisely $n$ CE points (the self intersection being elsewhere stable). In particular, $I_{0}$ is the space of all stable immersions in $\mathcal{A}$.

Let $\mathbb{G}$ be any Abelian group and let $f: I_{0} \rightarrow \mathbb{G}$ be an invariant, i.e. a function which is constant on each connected component of $I_{0}$. Given an immersion $i \in I_{n}$, a temporary co-orientation for $i$ is a choice of co-orientation at each of the $n \mathrm{CE}$ 
points $p_{1}, \ldots, p_{n}$ of $i$. Given a temporary co-orientation $\mathfrak{T}$ for $i$, define $f^{\mathfrak{T}}(i)$ as follows: Let $i_{1}, \ldots, i_{2^{n}}$ be the $2^{n}$ immersions in $I_{0}$ obtained from $i$ by slightly deforming it in the $2^{n}$ possible ways. We define:

$$
f^{\mathfrak{T}}(i)=\sum_{m=1}^{2^{n}} \epsilon_{1}^{m} \cdots \epsilon_{n}^{m} f\left(i_{m}\right)
$$

where $\epsilon_{k}^{m}$ is 1 or -1 according to whether in order to obtain $i_{m}$ we have deformed the configuration of $i$ at $p_{k}$ positively or negatively, with respect to $\mathfrak{T}$. The following is clear:

Lemma 3.1. If $i \in I_{n}$ and $\mathfrak{T}_{1}, \mathfrak{T}_{2}$ are two temporary co-orientations for $i$ which differ at precisely one CE of $i$ then $f^{\mathfrak{T}_{1}}(i)=-f^{\mathfrak{T}_{2}}(i)$.

By Lemma 3.1, the statement $f^{\mathfrak{T}}(i)=0$ is independent of the temporary co-orientation $\mathfrak{T}$ and we may simply write $f(i)=0$.

Definition 3.2. An invariant $f: I_{0} \rightarrow \mathbb{G}$ will be called of finite order if there is an $n$ such that $f(i)=0$ for all $i \in I_{n+1}$. The minimal such $n$ will be called the order of $f$.

Let $i: F \rightarrow \mathbb{R}^{3}$ be an immersion having a CE located at $p \in \mathbb{R}^{3}$. As in [N2], we define the degree $d_{p}(i)$ of the $\mathrm{CE}$ at $p$ as follows: Let $B$ be a tiny ball in $\mathbb{R}^{3}$ centered at $p . i^{-1}(B)$ is a union of some (two, three or four) discs in $F$ which pass $p$. Let $\hat{i}: F \rightarrow \partial B$ be the map obtained from $i$ as follows: On $F-i^{-1}(B)$ we define $\hat{i}$ by radial projection (centered at $p$ ). Now, if $D$ is one of the discs in $i^{-1}(B)$ then $i(D)$ cuts $\partial B$ into two hemispheres. $\hat{i}$ is defined to map $D$ onto the hemisphere which lies on the preferred side of $i(D)$ in $\mathbb{R}^{3}$. (Recall that $F$ is oriented). Finally we define $d_{p}(i)$ as the degree of the map $\hat{i}: F \rightarrow \partial B$ (the orientation on $\partial B$ being that induced from $B$ ).

Let $C_{p}(i)$ be the expression $R_{m}^{a}$ where $R^{a} \in \mathcal{C}$ is the symbol describing the configuration of the CE of $i$ at $p$ (one of the twelve symbols) and $m=d_{p}(i)$. Let $\mathcal{C}_{n}$ denote the set of all un-ordered $n$-tuples of expressions $R_{m}^{a}$ with $R^{a} \in \mathcal{C}, m \in \mathbb{Z}$. Finally, we define a map $C: I_{n} \rightarrow \mathcal{C}_{n}$ as follows: If $i \in I_{n}$ with CEs located at $p_{1}, \ldots, p_{n} \in \mathbb{R}^{3}$, then we define $C(i) \in \mathcal{C}_{n}$ to be the un-ordered $n$-tuple $\left[C_{p_{1}}(i), \ldots, C_{p_{n}}(i)\right]$. It is easy to see that $C: I_{n} \rightarrow \mathcal{C}_{n}$ is surjective.

Definition 3.3. A regular homotopy $H_{t}: F \rightarrow \mathbb{R}^{3}$ between immersions $i, j \in I_{n}$ will be called of type $\mathrm{B}$ if it is of the following form: If $B_{1}, \ldots, B_{n} \subseteq \mathbb{R}^{3}$ are little balls centered at the $n C E$ points of $i$ and $U=i^{-1}\left(\bigcup_{k} B_{k}\right)$ then $H_{t}$ fixes $U$ and moves $F-U$ within $\mathbb{R}^{3}-\bigcup_{k} B_{k}$.

Two immersions $i, j \in I_{n}$ will be called $\mathrm{AB}$ equivalent if there is a regular homotopy $H_{t}$ between $i$ and $j$ which is alternatingly of type $A$ and B. Such $a$ regular homotopy will be called an $\mathrm{AB}$ equivalence. 
A proof of the following proposition appears in [N2] for immersions including only quadruple points. The proof for the general case is identical, we include it here for completeness.

Proposition 3.4. Let $i, j \in I_{n}$, then $i$ and $j$ are $A B$ equivalent iff $C(i)=C(j)$.

Proof. If $i$ and $j$ are $\mathrm{AB}$ equivalent then clearly $C(i)=C(j)$. For the converse, assume $C(i)=C(j)$. One can order the CEs of $i$ and $j$, respectively $p_{1}^{\prime}, \ldots, p_{n}^{\prime}$ and $p_{1}, \ldots, p_{n}$, such that $C_{p_{k}^{\prime}}(i)=C_{p_{k}}(j), k=1, \ldots, n$. This means in particular, that if $B_{1}^{\prime}, \ldots, B_{n}^{\prime}$ and $B_{1}, \ldots, B_{n}$ are neighborhoods of the $p_{k}^{\prime} \mathrm{s}$ and $p_{k} \mathrm{~s}$ respectively, then for each $k$ there is an orientation preserving diffeomorphism from $B_{k}^{\prime}$ to $B_{k}$ which takes each sheet of $i(F) \cap B_{k}^{\prime}$ orientation preservingly onto the corresponding sheet of $j(F) \cap B_{k}$. These diffeomorphisms may all be realized by one ambient isotopy $U_{t}: \mathbb{R}^{3} \rightarrow \mathbb{R}^{3}$. There is then an isotopy $V_{t}: F \rightarrow F$ such that the final immersion $i^{\prime}$ of the regular homotopy $U_{t} \circ i \circ V_{t}$ satisfies that $i^{\prime}$ and $j$ have the same $n$ CE points $p_{1}, \ldots, p_{n} \in \mathbb{R}^{3}, i^{\prime-1}\left(\bigcup_{k} B_{k}\right)=j^{-1}\left(\bigcup_{k} B_{k}\right)$ which we name $U$, and $\left.i^{\prime}\right|_{U}=\left.j\right|_{U}$. Also $d_{p_{k}}\left(i^{\prime}\right)=d_{p_{k}}(j)$ for $k=1, \ldots, n$. Now $U$ is a union of some discs $D_{1}, \ldots, D_{r}$. We construct the following handle decomposition of $F . D_{1}, \ldots, D_{r}$ will be the 0 -handles. If $g$ is the genus of $F$ we will have 1-handles $h_{1}, \ldots, h_{2 g+r-1}$ as follows: $h_{1}, \ldots, h_{2 g}$ will each have both ends glued to $D_{1}$ such that $F-\left(D_{1} \cup h_{1} \cup \cdots \cup h_{2 g}\right)$ will be a disc containing $D_{2}, \ldots, D_{r}$. Then for $k=1, \ldots r-1, h_{2 g+k}$ will have one end glued to $D_{k}$ and the other to $D_{k+1}$. The complement of all 0- and 1-handles is again one disc, which will be the unique 2-handle. We will now construct a regular homotopy of the form $i^{\prime} \circ V_{t}^{\prime}$ ( $V_{t}^{\prime}: F \rightarrow F$ an isotopy) from $i^{\prime}$ to an immersion $i^{\prime \prime}$ which will have the property that the restrictions of $i^{\prime \prime}$ and $j$ to all 1-handles, are regularly homotopic keeping all 0 -handles fixed. Since $i^{\prime}$ and $j$ are regularly homotopic (recall $i, j \in I_{n} \subseteq \mathcal{A}$ ), this is already true for $h_{1}, \ldots, h_{2 g}$. Now take $h_{2 g+1}$. If $\left.i^{\prime}\right|_{h_{2 g+1}}$ and $\left.j\right|_{h_{2 g+1}}$ are not regularly homotopic keeping $D_{1}$ and $D_{2}$ fixed, then $V_{t}^{\prime}$ performs one full rotation of $D_{2}$, creating a Dehn twist in a thin annulus around $D_{2}$ in $F \cdot h_{2 g+1}$ will now satisfy the needed property. Note also that this rotation of $D_{2}$ moves only $h_{2 g+1}$ and $h_{2 g+2}$, keeping all other 0 - and 1-handles fixed. We continue this way along the chain of 1-handles, rotating $D_{k+1}$ if necessary for the sake of $h_{2 g+k}$. For $k<r-1$ this will also move $h_{2 g+k+1}$, but we never need to move 1-handles that have previously been taken care of. Also $d_{p_{k}}\left(i^{\prime \prime}\right)=d_{p_{k}}(i)=d_{p_{k}}(j)$ for all $k=1, \ldots, n$. We now perform a regular homotopy $H_{t}$ on the union of 0- and 1-handles which fixes the 0 -handles, and regularly homotopes each 1-handle $h$, from $\left.i^{\prime \prime}\right|_{h}$ to $\left.j\right|_{h}$, avoiding $\bigcup_{k} B_{k}$. This is possible by the construction of $i^{\prime \prime}$. Denote our 2-handle by $D$. So far we have constructed $H_{t}$ only on $F-D$. By means of [S], $H_{t}$ may be extended to $D$, still avoiding $\bigcup_{k} B_{k}$, arriving at an immersion $i^{\prime \prime \prime}$. And so, we are left with regularly homotoping $\left.i^{\prime \prime \prime}\right|_{D}$ to $\left.j\right|_{D}($ relative $\partial D)$. Since $d_{p_{k}}\left(i^{\prime \prime \prime}\right)=d_{p_{k}}(j)$ for all $k=1, \ldots, n$, these maps are homotopic in $\mathbb{R}^{3}-\bigcup_{k} B_{k}$. It then follows from the Smale-Hirsch Theorem $([\mathrm{H}])$, that they are also regularly homotopic in $\mathbb{R}^{3}-\bigcup_{k} B_{k}$ (since the obstruction to that would lie in $\pi_{2}\left(\mathrm{SO}_{3}\right)=0$ ). 
The regular homotopy from $i$ to $i^{\prime \prime}$ was of type A, and that from $i^{\prime \prime}$ to $j$ was of type B.

Proposition 3.5. Let $f: I_{0} \rightarrow \mathbb{G}$ be an invariant of order $n$. Let $i \in I_{n}$ be an immersion with CEs $p_{1}, \ldots, p_{n}$ and assume the configuration of $i$ at $p_{1}$ is of type $H^{1}$ or $Q^{2}$. Let $\mathfrak{T}$ be a temporary co-orientation for $i$. Then the element $a=f^{\mathfrak{T}}(i) \in \mathbb{G}$ satisfies $a=-a$ (or equivalently $2 a=0$ ).

Proof. For $k=1, \ldots, n$ let $B_{k}$ be a small neighborhood of $p_{k}$ in $\mathbb{R}^{3}$. Let $h$ : $B_{1} \rightarrow B_{1}$ be the diffeomorphism given by Proposition 2.2. We use $h$ on $B_{1}$ and the identity map on $B_{2}, \ldots, B_{n}$, in the proof of Proposition 3.4, getting an $\mathrm{AB}$ equivalence $H_{t}$ from $i$ to itself which reverses the co-orientation of the configuration in $B_{1}$ and fixes $F$ in $B_{2}, \ldots, B_{n}$. Let $\mathfrak{T}_{t}$ be the temporary co-orientation for $H_{t}$ which is obtained by continuously carrying $\mathfrak{T}$ along the regular homotopy. If at some time $t_{0}, H_{t}$ passes through an $(n+1)$ th $\mathrm{CE}$ at $p \in \mathbb{R}^{3}$, we choose an arbitrary co-orientation for $H_{t_{0}}$ at $p$. Together with $\mathfrak{T}_{t_{0}}$ this gives a temporary coorientation $\mathfrak{T}^{\prime}$ for the $n+1$ CEs of $H_{t_{0}}$. Since $f$ is of order $n, f^{\mathfrak{T}^{\prime}}\left(H_{t_{0}}\right)=0$ which means that $f^{\mathfrak{T}_{t_{0}-\epsilon}}\left(H_{t_{0}-\epsilon}\right)=f^{\mathfrak{T}_{t_{0}+\epsilon}}\left(H_{t_{0}+\epsilon}\right)$. Finally we arrive back at $i$ but with opposite co-orientation than the original one at $p_{1}$ and the same co-orientation at $p_{2}, \ldots, p_{n}$.

By the string of equalities $f^{\mathfrak{T}_{t_{0}-\epsilon}}\left(H_{t_{0}-\epsilon}\right)=f^{\mathfrak{T}_{t_{0}+\epsilon}}\left(H_{t_{0}+\epsilon}\right)$ on the one hand and by Lemma 3.1 on the other hand, we finally get $f^{\mathfrak{T}}(i)=-f^{\mathfrak{T}}(i)$.

Corollary 3.6. Let $f: I_{0} \rightarrow \mathbb{G}$ be an invariant of order $n$. Let $i \in I_{n}$ be an immersion with at least one CE of type $H^{1}$ or $Q^{2}$. Then $f^{\mathfrak{T}}(i)$ is independent of the temporary co-orientation $\mathfrak{T}$.

Proof. This follows from Lemma 3.1 and Proposition 3.5

Remark 3.7. We have seen in the proof of Proposition 3.5 that if $i \in I_{n}$ has a CE of type $H^{1}$ or $Q^{2}$ then there is an $\mathrm{AB}$ equivalence from $i$ to itself such that if we follow that $\mathrm{CE}$ along the $\mathrm{AB}$ equivalence, then it returns to itself but with opposite co-orientation. If one seeks a globally defined co-orientation, it is a minimal requirement that it be continuously defined along $\mathrm{AB}$ equivalences. So we see that such globally defined co-orientation does not exist for CEs of type $H^{1}$ and $Q^{2}$.

If $f$ is an invariant of order $n$ then Corollary 3.6 enables us to extend $f$ to $I_{n}$ as follows: We choose once and for all a permanent co-orientation for the ten configurations which allow it; in fact, we choose those co-orientations given in Section 2 above. Now, if $i \in I_{n}$ and all $n$ CEs of $i$ are not of configuration $H^{1}$ or $Q^{2}$ then we define $f(i)$ using our permanent co-orientation for each of the CEs. If at least one of the CEs of $i$ is of configuration $H^{1}$ or $Q^{2}$ then by Corollary 3.6 $f(i)$ is well defined, independent of a temporary co-orientation. We will assume from now on without mention that any $f$ of order $n$ is extended to $I_{n}$ in this way. (Note that if $f$ is of order $n$ then we are not extending $f$ to $I_{k}$ for $0<k<n$ ). 
Proposition 3.8. Let $f$ be an invariant of order $n$ and $i, j \in I_{n}$. If $C(i)=C(j)$ then $f(i)=f(j)$.

Proof. By Proposition $3.4 i$ and $j$ are AB equivalent. As in the proof of Proposition $3.5, f$ is unchanged whenever we pass an $(n+1)$ th CE and so $f(i)=f(j)$.

By Proposition 3.8 and since $C: I_{n} \rightarrow \mathcal{C}_{n}$ is surjective, any order $n$ invariant $f$ induces a well defined function $u(f): \mathcal{C}_{n} \rightarrow \mathbb{G}$. Now $u(f)=u(g)$ iff $f$ and $g$ differ by an invariant of order at most $n-1$. And so if $V_{n}$ denotes the group of all invariants on $\mathcal{A}$ of order at most $n$ then $f \mapsto u(f)$ induces an injection $u: V_{n} / V_{n-1} \rightarrow \mathcal{C}_{n}^{*}$ where $\mathcal{C}_{n}^{*}$ is the group of all functions from $\mathcal{C}_{n}$ to $\mathbb{G}$. Finding the image of $u$ for all $n$ would give a classification of all finite order invariants. A first relation that must be satisfied by the image of $u$ follows from Proposition 3.5 above, namely:

Corollary 3.9. If $g \in \mathcal{C}_{n}^{*}$ is in the image of $u$ and if $x \in \mathcal{C}_{n}$ involves the symbol $H^{1}$ or $Q^{2}$ then $2 g(x)=0$.

The purpose of the next section is to specify a subgroup $\Delta_{n} \subseteq \mathcal{C}_{n}^{*}$ which contains the image of $u . \Delta_{n}$ will be the subgroup of $\mathcal{C}_{n}^{*}$ which is determined by the relation of Corollary 3.9 above and by all relations obtained by looking at local 2-parameter families of immersions.

In Section 5 we will show that $u: V_{1} / V_{0} \rightarrow \Delta_{1}$ is surjective. By this we classify all order one invariants (Theorem 5.1). We will show in Section 7 that for $\mathbb{G}=\mathbb{Z} / 2$ and $n>1, u: V_{n} / V_{n-1} \rightarrow \Delta_{n}$ is not surjective. This we do by demonstrating one particular function in $\Delta_{n}$ which is not attained by $u$. We will also show that for any $\mathbb{G}$ there exist invariants of any order $n$.

Question 3.10. What is the image of $u$ for $n>1$ ? (given $F, \mathcal{A}$ and $\mathbb{G}$ ).

We now explain our analogy with knot theory referred to in the introduction. The space $I_{0}$ on which we have defined our invariants is the space of all stable immersions of $F$ into $\mathbb{R}^{3}$. For the circle $S^{1}$, a stable immersion into $\mathbb{R}^{3}$ is necessarily an embedding i.e. a knot, thus our finite order invariants of immersions are analogous to those of knots. The $\operatorname{set} \mathcal{C}_{n}$ that we have defined above is analogous to the set of all chord diagrams of order $n$. The analogy is made clear by Propositions 3.4 and 3.8 above. $\Delta_{n}$ which we define below will be analogous to the space of functions on chord diagrams which satisfy the 1-term and 4-term relations. (More precisely, our definition of $\Delta_{n}$ will only use 2-parameter families of immersions, whereas the 1-term relation of knot theory is produced by an essential loop in $\operatorname{Imm}\left(S^{1}, \mathbb{R}^{3}\right)$; but by Remark 5.2 below, the analogous essential loops in our setting produce no additional relations). An analogue of our relation of Corollary 3.9 above, does not exist in knot theory, since the strata of knot theory are globally two sided. Note also that the relation of Corollary 3.9 is obtained from a loop in $I_{n} \cup I_{n+1}$ whereas all our other relations, (as the relations of knot theory), will 
be obtained from loops in $I_{n-1} \cup I_{n}$. Finally, the analogue for knots of our Question 3.10 has been fully answered in $[\mathrm{K}]$ for $\mathbb{G}=\mathbb{Q}$, namely, the analogue of $u: V_{n} / V_{n-1} \rightarrow \Delta_{n}$ is surjective for all $n$.

At this point we would like to further justify our definition of finite order invariants (Definition 3.2). For an invariant to be of order $n$, its values in a neighborhood of any immersion with $n+1$ CEs must satisfy a certain restriction; whereas, no restriction is imposed on the invariant in a neighborhood of other, more general immersions of codim $n+1$. If one wishes to impose such additional restrictions, then one must first note that in general, the stratification of $\operatorname{Imm}\left(F, \mathbb{R}^{3}\right)$ in a neighborhood of an immersion of codim $n+1$ does not resemble that of an immersion with $n+1$ CEs. And so additional restrictions related to general $\operatorname{codim} n+1$ immersions would require separate definitions suited to the structure of the stratification in each case. Furthermore, if we wish to follow the example set by knot theory, we should note the following: Consider an immersion of $S^{1}$ in $\mathbb{R}^{3}$ which has a triple point, i.e. self intersection equivalent to the intersection of the three coordinate axes in $\mathbb{R}^{3}$. This is an immersion of codim 3 and the stratification of $\operatorname{Imm}\left(S^{1}, \mathbb{R}^{3}\right)$ in a neighborhood of such immersion happens to be identical to that in a neighborhood of an immersion with three double points, namely, three hypersurfaces intersecting in general position. So, one might want to impose further restrictions on knot invariants of order $n$ using codim $n+1$ immersions which include such triple point and $n-2$ double points, in a way identical to that which uses immersions with $n+1$ double points. Such additional restriction would diminish the space of invariants of order at most $n$. (In fact, it is easy to show that the space of knot invariants of order at most $n$ with this restricted definition is equal to the space of invariants of order at most $n-1$ with the usual definition.) We conclude that the option of adding another restriction naturally presents itself in the setting of knot theory, but knot theorists have chosen not to do so. By this choice they have established the meaning of finite order invariants as being a restriction related to simplest (i.e. codim 1) singularities occurring in several distinct places, and not to other, more complicated singularities.

\section{Local analysis}

Let $i \in \mathcal{A}$ be an immersion with a self intersection of local codim 2 at $p$ and $n-1$ additional self-intersections of local codim 1 (i.e. CEs) at $p_{1}, \ldots, p_{n-1}$. We look at a 2-parameter family of immersions which moves $F$ only in a neighborhood of $p$, such that the immersion $i$ corresponds to parameters $(0,0)$ and such that this 2 parameter family is transverse to the local codim 2 stratum at $i$. In this 2-parameter family of immersions we look at a loop which encircles the point of intersection with the codim 2 strata, i.e. a circle around the origin in the parameter plane. This circle crosses the local codim 1 strata some $r$ times. Between each two intersections we have an immersion in $I_{n-1}$ with the same $n-1 \mathrm{CEs}$, at $p_{1}, \ldots, p_{n-1}$. At 
each intersection with the local codim 1 strata, an $n$th CE is added, obtaining an immersion in $I_{n}$. Let $i_{1}, \ldots, i_{r}$ be the $r$ immersions in $I_{n}$ so obtained and let $\epsilon_{k}$, $k=1, \ldots, r$ be 1 or -1 according to whether we are passing the $n$th CE of $i_{k}$ in the direction of its permanent co-orientation, if it has one. If the CE is of type $H^{1}$ or $Q^{2}$ then $\epsilon_{k}$ is arbitrarily chosen. Now let $f: I_{0} \rightarrow \mathbb{G}$ be an invariant of order $n$, then it is easy to see that $\sum_{k=1}^{r} \epsilon_{k} f\left(i_{k}\right)=0$. (Note that by Corollary 3.9 the arbitrariness of the choice of $\epsilon_{k}$ for types $H^{1}$ and $Q^{2}$ has no effect). Looking now at $u: V_{n} / V_{n-1} \rightarrow \mathcal{C}_{n}^{*}$ we obtain relations that must be satisfied by a function in $\mathcal{C}_{n}^{*}$ in order for it to lie in the image of $u$. In this section we will find all relations on $\mathcal{C}_{n}^{*}$ obtained in this way. The relations on a $g \in \mathcal{C}_{n}^{*}$ will be written as relations on the symbols $R_{m}^{a}$, e.g. $0=T_{m}^{a}-T_{m}^{3-a}$ will stand for the set of all relations of the form $0=g\left(\left[T_{m}^{a}, R_{2} a_{d_{2}}, \ldots, R_{n_{d_{n}}}^{a_{2}}\right]\right)-g\left(\left[T_{m}^{3-a}, R_{d_{2}}^{a_{2}}, \ldots, R_{n_{d_{n}}}^{a_{n}}\right]\right)$ with arbitrary $R_{2}^{a} d_{2}, \ldots, R_{n_{d_{n}}}^{a_{n}}$. Thus the relations of Corollary 3.9 are written as $0=2 H_{m}^{1}$ and $0=2 Q_{m}^{2}$ (for all $m$ ).

In this section we will use our extended set of symbols $\widetilde{\mathcal{C}}$ and we denote by $\widetilde{\mathcal{C}_{n}}$ the set of all un-ordered $n$-tuples of expressions of the form $R_{m}^{a}$ where $R^{a} \in \widetilde{\mathcal{C}}$. Recalling Lemma 3.1, we identify $\mathcal{C}_{n}^{*}$ with the group of functions on $\widetilde{\mathcal{C}}_{n}$ which satisfy the relations $H_{m}^{0}=-H_{m}^{2}, Q_{m}^{0}=-Q_{m}^{4}, Q_{m}^{1}=-Q_{m}^{3}$ (these represent relations in the above sense).

We now look at local 2-parameter families of immersions which are transverse to the various local codim 2 strata. These may be divided into six types (see [HK]) which we will name after the types of CEs appearing in the 2-parameter family: $E H, T T, E T, H T, T Q, Q Q$. In the notation of [HK] they are respectively: $A_{0}^{2}\left|A_{2}, A_{0}^{3}\right| A_{2},\left(A_{0}^{2} \mid A_{1}^{+}\right)\left(A_{0}\right),\left(A_{0}^{2} \mid A_{1}^{-}\right)\left(A_{0}\right),\left(A_{0}^{3} \mid A_{1}\right)\left(A_{0}\right), A_{0}^{5}$.

For each of the first five types we give the following:

1. Formula for a local representative.

2. Sketch of the configuration for some value $\left(\lambda_{1}, \lambda_{2}\right)$ of the parameters.

3. Diagram of the 2 dimensional parameter space, where intersection with the codim 1 strata is depicted, including their co-orientations (this is called a bifurcation diagram).

4. The relation arising.

Note that Corollary 3.9 takes care of the cases when there is actually no co-orientation.

For these five types, the bifurcation diagram is obtained from the sketch and formula in a straight forward manner. Whenever the plane $x=0$ appears in a configuration below, we assume (by rotating the configuration if necessary) that its preferred side is $x>0$. The integer $m$ in terms of which the degrees of the CEs are given, is the degree of the central codim 2 immersion at its codim 2 self intersection. (We have originally defined degree only for CEs, but the same definition applies to any self-intersection). We then go on to type $Q Q$; it requires special analysis which will be done in detail. 

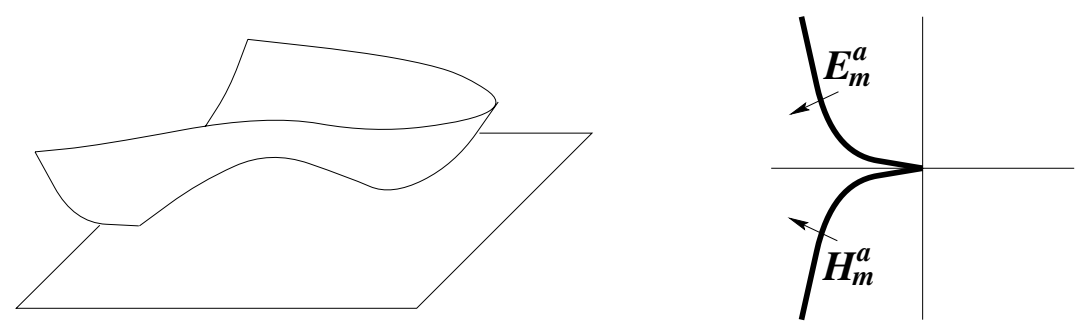

Fig. 1. $E H$ configuration

$E H: \quad z=0, \quad z=y^{2}+x^{3}+\lambda_{1} x+\lambda_{2}$.

$$
0=E_{m}^{a}-H_{m}^{a}
$$
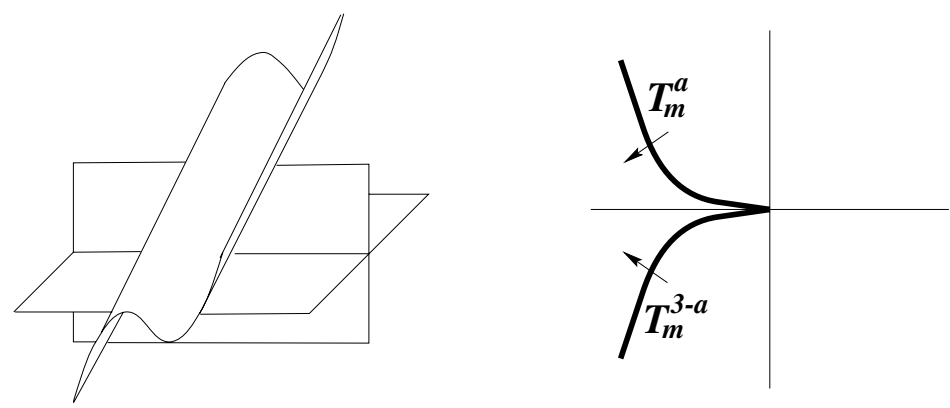

Fig. 2. $T T$ configuration

$T T: \quad z=0, \quad y=0, \quad z=y+x^{3}+\lambda_{1} x+\lambda_{2}$.

$$
0=T_{m}^{a}-T_{m}^{3-a}
$$
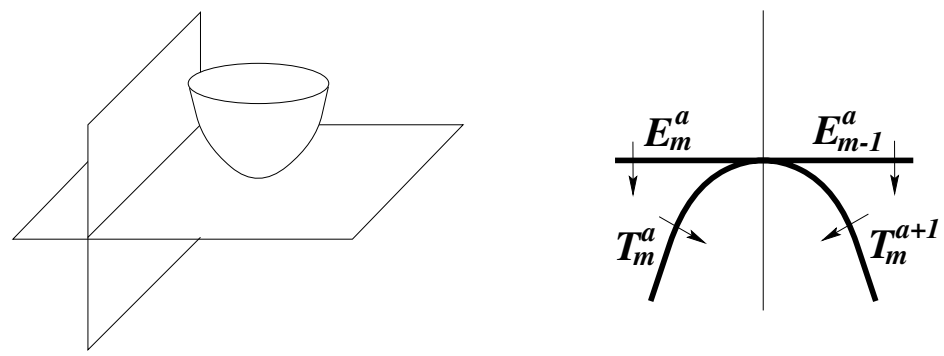

Fig. 3. ET configuration 
$E T: \quad z=0, \quad x=0, \quad z=\left(x-\lambda_{1}\right)^{2}+y^{2}+\lambda_{2}$.

$$
0=T_{m}^{a}-T_{m}^{a+1}-E_{m-1}^{a}+E_{m}^{a}
$$
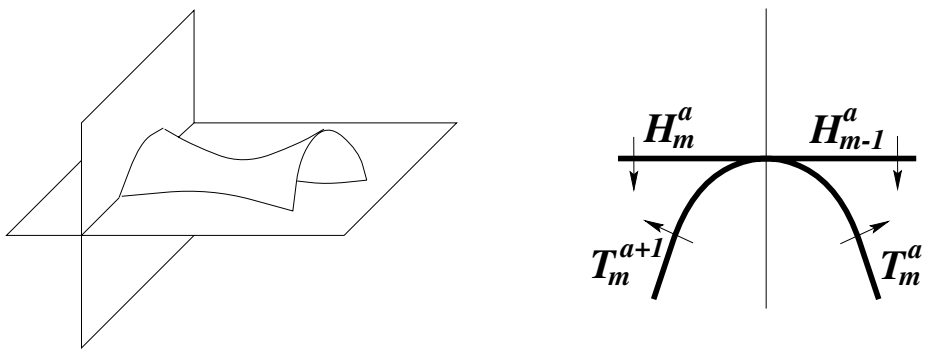

Fig. 4. $H T$ configuration

$H T: \quad z=0, \quad x=0, \quad z=\left(x-\lambda_{1}\right)^{2}-y^{2}+\lambda_{2}$.

$$
0=-T_{m}^{a+1}+T_{m}^{a}-H_{m-1}^{a}+H_{m}^{a}
$$
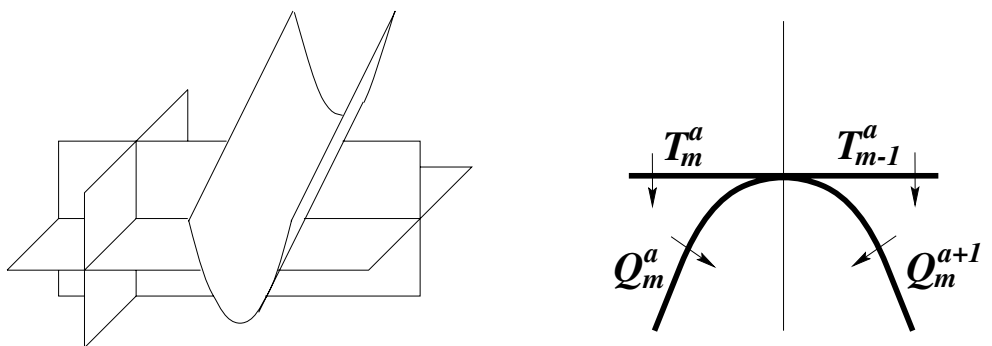

Fig. 5. $T Q$ configuration

$T Q: \quad z=0, \quad y=0, \quad x=0, \quad z=y+\left(x-\lambda_{1}\right)^{2}+\lambda_{2}$.

$$
0=Q_{m}^{a}-Q_{m}^{a+1}-T_{m-1}^{a}+T_{m}^{a}
$$

$Q Q$ : This configuration is a quintuple point, i.e. five sheets passing through a point, any three of which are in general position. We construct a 2-parameter family of quintuples of oriented planes which represents a local 2-parameter family of immersions which is transverse to the local quintuple point stratum. Let $P_{1}, \ldots, P_{5}$ be five oriented planes through the origin in $\mathbb{R}^{3}$ any three of which are in general position. For $k=1, \ldots, 5$ let $u_{k} \in \mathbb{R}^{3}$ be the unit vector which is perpendicular to $P_{k}$ and pointing into the preferred side of $P_{k}$ in $\mathbb{R}^{3}$. Any three of 
the vectors $u_{1}, \ldots, u_{5}$ are independent. A vector $\left(\lambda_{1}, \ldots, \lambda_{5}\right) \in \mathbb{R}^{5}$ will represent the quintuple of planes $P_{1}^{\lambda_{1}}, \ldots, P_{5}^{\lambda_{5}}$ in $\mathbb{R}^{3}$ where $P_{k}^{\lambda}=\left\{x \in \mathbb{R}^{3}: x \cdot u_{k}=\lambda\right\}$. (In particular, for each $k: P_{k}^{0}=P_{k}$ ).

If $\lambda \in \mathbb{R}$ and $v \in \mathbb{R}^{3}$ then $P_{k}^{\lambda+v \cdot u_{k}}$ is the translate of the plane $P_{k}^{\lambda}$ by the vector $v$. Let $V \subseteq \mathbb{R}^{5}$ be the 3-dimensional subspace defined by $V=\left\{\left(v \cdot u_{1}, \ldots, v\right.\right.$. $\left.\left.u_{5}\right): v \in \mathbb{R}^{3}\right\}$ then $\left(\lambda_{1}, \ldots, \lambda_{5}\right) \in V$ iff $P_{1}^{\lambda_{1}}, \ldots, P_{5}^{\lambda_{5}}$ all meet at a point. Let $U=V^{\perp} \subseteq \mathbb{R}^{5}$ then $U$ represents a local 2-parameter family of immersions which is transverse to the local codim 2 stratum of quintuple points. If $A$ is the $5 \times 3$ matrix whose rows are $u_{1}, \ldots, u_{5}$ then the columns of $A$ span $V$ and so $U=V^{\perp}$ is the left kernel of $A$ i.e. $U=\left\{\left(\lambda_{1}, \ldots, \lambda_{5}\right): \lambda_{1} u_{1}+\cdots+\lambda_{5} u_{5}=0\right\}$.

We now find the points in $U$ which represent configurations involving a CE, which in this case must be a quadruple point. Let $l_{k}=U \cap\left(V+\mathbb{R} \epsilon_{k}\right)$ where $\epsilon_{1}, \ldots, \epsilon_{5}$ is the standard basis of $\mathbb{R}^{5}$ (i.e. $\epsilon_{1}=(1,0,0,0,0)$ etc.). Then $l_{k}$ represents all configurations in $U$ where the planes $\left\{P_{j}: j \neq k\right\}$ meet at a quadruple point; indeed, an element of $l_{k}$ is an element of $U$ which is obtained from an element of $V$ i.e. a quintuple point, by adding $r \epsilon_{k}$ for some $r$ i.e. pushing away the plane $P_{k}$.

For a given $l_{k}$ we would like to determine the configuration of the quadruple point represented by it. Take say $l_{5}$. We must look at the configuration of the four planes $P_{1}^{\lambda_{1}}, \ldots, P_{4}^{\lambda_{4}}$ given by points $\left(\lambda_{1}, \ldots, \lambda_{5}\right)$ on the two sides of $l_{5}$ in $U$, and see how many of the planes $P_{1}^{\lambda_{1}}, \ldots, P_{4}^{\lambda_{4}}$ have the simplex created by them, on their non-preferred side. It is enough to check one point on each side of $l_{5}$ in $U$ and as we shall see, it will be most convenient to look at the points of $l_{5}^{\perp}$ (where $\perp$ here means the orthogonal complement in $U$ ). Now $l_{5}^{\perp}=\epsilon_{5}^{\perp} \cap U$ (the $\perp$ on the right is the orthogonal complement in $\mathbb{R}^{5}$ ); i.e. $l_{5}^{\perp}=\left\{\left(\lambda_{1}, \ldots, \lambda_{4}, 0\right) \in \mathbb{R}^{5}: \lambda_{1} u_{1}+\cdots+\lambda_{4} u_{4}=0\right\}$. For such points we can determine the configuration of the simplex using the following lemma:

Lemma 4.1. Let $v_{1}, \ldots, v_{4} \in \mathbb{R}^{3}$ be four vectors such that any three of them are independent and $v_{1}+\cdots+v_{4}=0$. If $0<\mu_{1}, \ldots, 0<\mu_{4} \in \mathbb{R}$ then the origin $0 \in \mathbb{R}^{3}$ lies in the interior of the simplex determined by the four planes $\left\{x \in \mathbb{R}^{3}: x \cdot v_{k}=\mu_{k}\right\}$.

Proof. Since $0 \cdot v_{k}=0<\mu_{k}$, the domain determined by the four planes in which the origin lies is: $D=\left\{x \in \mathbb{R}^{3}: x \cdot v_{k} \leq \mu_{k}\right.$ for all $\left.1 \leq k \leq 4\right\}$. This is a convex domain in $\mathbb{R}^{3}$. If it is not the simplex determined by the four planes then it is unbounded and so there is a ray based at 0 which is contained in $D$, i.e. there is a vector $v \neq 0$ such that $r v \cdot v_{k} \leq \mu_{k}$ for every $1 \leq k \leq 4$ and any $r>0$. It follows that for each $k: v \cdot v_{k} \leq 0$. If for some $k, v \cdot v_{k}<0$ then $v \cdot\left(v_{1}+\cdots+v_{4}\right)<0$ contradicting $v_{1}+\cdots+v_{4}=0$. So $v \cdot v_{k}=0$ for all $k$, contradicting the fact that any three of $v_{1}, \ldots, v_{4}$ are independent.

Back to our $l_{5}^{\perp}$, we use Lemma 4.1 with $v_{k}=\lambda_{k} u_{k}$ and $\mu_{k}=\left(\lambda_{k}\right)^{2}, k=1, \ldots, 4$, (note that $\lambda_{1}, \ldots, \lambda_{4}$ are all non-zero since each three $u_{k}$ s are independent) 
obtaining that 0 lies in the interior of the simplex determined by the equations $x \cdot \lambda_{k} u_{k}=\left(\lambda_{k}\right)^{2}(k=1, \ldots, 4)$ which is the same as $x \cdot u_{k}=\lambda_{k}$ i.e. the planes $P_{1}^{\lambda_{1}}, \ldots, P_{4}^{\lambda_{4}}$. Now, the vector $u_{k}$, when based at $P_{k}^{\lambda_{k}}$, points into the preferred side of $P_{k}^{\lambda_{k}}$ in $\mathbb{R}^{3}$. On the other hand, $u_{k}$ points away from the side where the origin lies (which is the side where the simplex lies) iff $\lambda_{k}>0$. We conclude that if $\left(\lambda_{1}, \ldots, \lambda_{4}, 0\right) \in l_{5}^{\perp}$ then the number of faces of the simplex determined by $P_{1}^{\lambda_{1}}, \ldots, P_{4}^{\lambda_{4}}$ which have the simplex on their non-preferred side, is precisely the number of positive numbers among $\lambda_{1}, \ldots, \lambda_{4}$.

The origin of $U$ splits $l_{5}^{\perp}$ into two half lines. Clearly the number $p$ of plus signs is constant on such a half-line and is $4-p$ on the other half line. So for given quintuple point, our task is to find the number $p$ of plus signs in each of the ten half lines of the $l_{k}^{\perp}$ s. Now $l_{k}^{\perp}$ has $\lambda_{k}=0$, so it partitions $U$ into the domains where $\lambda_{k}>0$ and $\lambda_{k}<0$. We thus use it to determine the sign of $\lambda_{k}$ in the half-lines of $l_{j}^{\perp}$ for $j \neq k$. Examples of such analysis appear in Figure 6, which we now explain: Each of the short thick lines in a diagram represents an $l_{k}^{\perp}$. The arrows on each $l_{k}^{\perp}$ point to the side of it in $U$ where $\lambda_{k}$ is positive. To determine the number $p$ of positive $\lambda_{j}$ s corresponding to a given half line of $l_{k}^{\perp}$ we need to count for how many $l_{j}^{\perp} \mathrm{s}(j \neq k)$ this half line lies on their positive side, i.e. the side designated by the arrows. This is the number appearing in the circle located on the given half $l_{k}^{\perp}$ in the diagram (at the tip of the short thick line). Finally the longer thinner lines are the $l_{k}$ s themselves (each drawn perpendicular to the corresponding $l_{k}^{\perp}$ ). The pair of numbers at each tip of $l_{k}$ is simply copied from the corresponding sides of $l_{k}^{\perp}$. When passing an $l_{k}$, this pair of numbers (which is of the form $p, 4-p$ ) tells us the type of quadruple point we are passing, and the co-orientation with which we are passing it, namely, if say we use symbols only in $\mathcal{C}$ then the side chosen by our permanent co-orientation is the side where the larger number of the pair appears. (As before, Corollary 3.9 takes care of the case when there is no co-orientation).

Finally we need to determine the degrees $d_{p}(i)$ at each of the ten quadruple points. Let $m$ be the degree of the quintuple point and look say at $l_{1}$. We claim that the half of $l_{1}$ which has $\lambda_{1}>0$ represents quadruple points with degree $m$ whereas the half with $\lambda_{1}<0$ represents quadruple points with degree $m-1$. To establish this we need to show that a quadruple point represented by a point in the half of $l_{1}$ with $\lambda_{1}>0$ is obtained from a translate of our quintuple point by pushing $P_{1}$ into its preferred side i.e. the side pointed at by $u_{1}$. Recall that a point $\left(\lambda_{1}, \ldots, \lambda_{5}\right) \in l_{1}$ is of the form $v+r \epsilon_{1}$ where $v$ is perpendicular to $U$ and represents a common translation of all five planes. So $P_{1}^{\lambda_{1}}$ is obtained by pushing $P_{1}$ away from the quintuple point represented by $v$, and this push is into the preferred side of $P_{1}$ iff $r>0$. It remains to notice that the sign of $\lambda_{1}$ is the same as that of $r$, since $\left(\lambda_{1}, \ldots, \lambda_{5}\right)$ is the orthogonal projection of $(r, 0,0,0,0)$ to $U$. The integers $m$ or $m-1$ appearing at the tips of each $l_{k}$ in the diagrams are the degrees. (Recall that the arrows on $l_{k}^{\perp}$ point to the side where $\lambda_{k}>0$ ). 

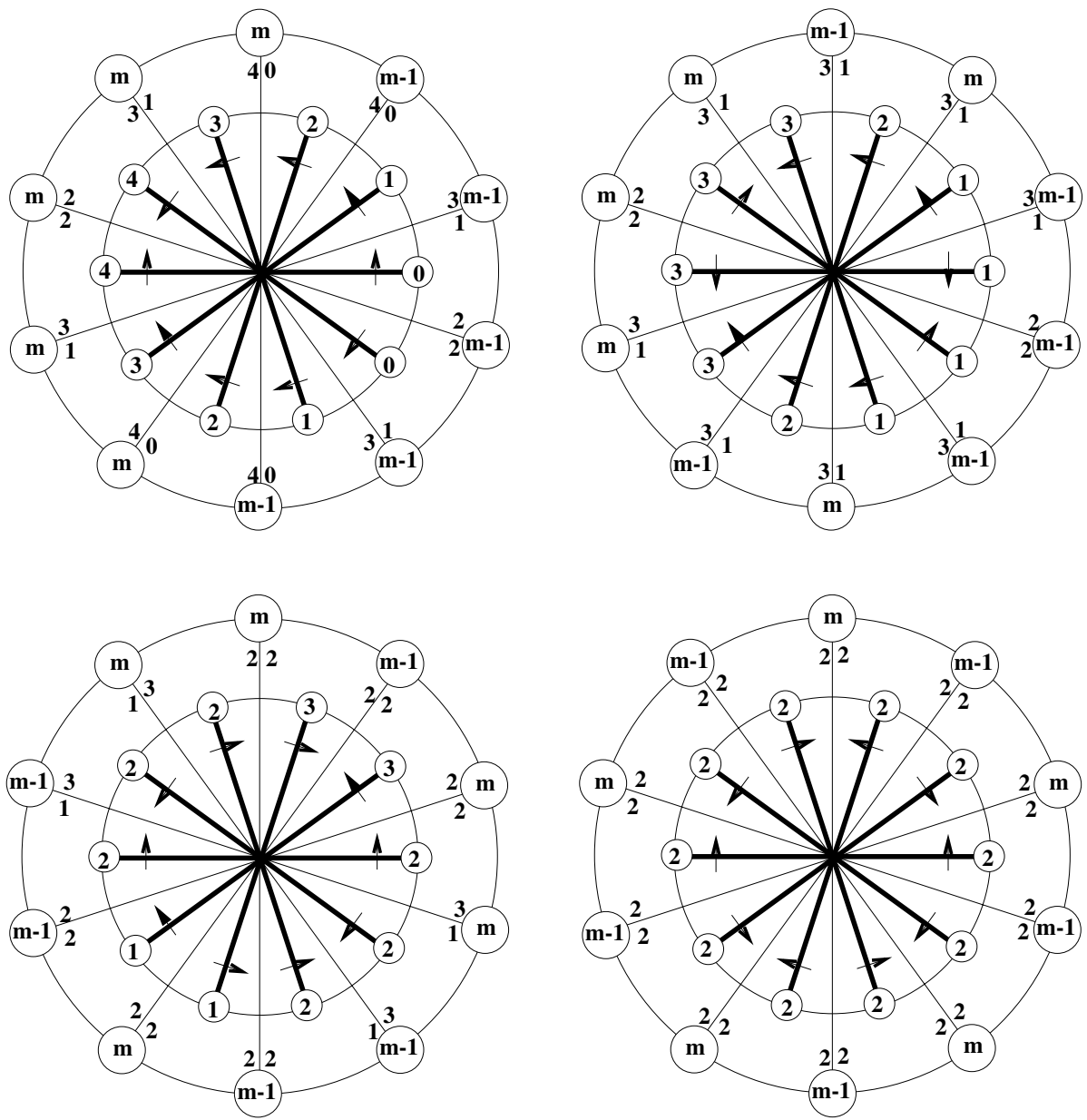

Fig. 6. $Q Q$ configuration

Once we have all this information registered at the tips of the $l_{k} \mathrm{~s}$, we can read off the diagram the relation determined by the given quintuple point. We claim that for any configuration of lines and arrows as above, eight of the terms cancel, always leaving the same relation:

$$
Q_{m}^{2}=Q_{m-1}^{2}
$$

We offer two ways of seeing this: The first way is to verify that the four diagrams appearing in Figures 6 are in fact all essentially distinct ways for choosing such distribution of arrows. Then explicitly write out the relation obtained by each of them. We only remark that there is no loss of generality by the fact that the diagrams are sketched with equal angles between the $l_{k} \mathrm{~s}$, since all that is relevant 
to us is the cyclic ordering of the $l_{k}^{\perp} \mathrm{s}$ and not the common cyclic ordering of all $l_{1}, \ldots, l_{5}, l_{1}^{\perp}, \ldots, l_{5}^{\perp}$.

A second way to see that the same relation $Q_{m}^{2}=Q_{m-1}^{2}$ is always obtained is as follows: Since we have five arrows pointing clockwise and five counter-clockwise, there must be two consecutive arrows which are pointing toward each other. These half $l_{k}^{\perp}$ s must have the same number at their tip, since they are on the same side of any other $l_{j}^{\perp}$ and are both on the positive side of each other (i.e. the side designated by the arrow). We now look at the corresponding positive half $l_{k} \mathrm{~s}$. They both have degree $m$ and will both have the same number copied next to them from the half $l_{k}^{\perp}$, but written on the opposite side. So their contribution to the relation is precisely the negative of each other. The same will be true for the negative halves of these $l_{k}$ s (only now with degrees $m-1$ ). We now erase these two lines from the diagram and argue by (a two step) induction that the formal property analogous to the one we are proving, holds for the remaining three line diagram (checking that it holds for a one line diagram). It remains to observe that once we re-insert the two erased lines, they jointly add precisely 1 to the numbers at the tips of all other short thick lines and so the property continues to hold for the five line diagram.

We denote by $\Delta_{n}=\Delta_{n}(\mathbb{G})$ the subgroup of $\mathcal{C}_{n}^{*}$ satisfying relations 1-6 above and the relation of Corollary 3.9, namely: $0=2 H_{m}^{1}=2 Q_{m}^{2}$. Recall also that since we are identifying $\mathcal{C}_{n}^{*}$ with a set of functions on $\widetilde{\mathcal{C}}_{n}$, we also have the relations $H_{m}^{0}=-H_{m}^{2}$ and $Q_{m}^{a}=-Q_{m}^{4-a}(a=0,1)$.

Now that we have obtained our relations, we return to our original $\mathcal{C}_{n}$ with only twelve symbols i.e. we dispose of the redundant symbols $H^{0}, Q^{0}, Q^{1}$. Let $\mathbb{B} \subseteq \mathbb{G}$ be defined by $\mathbb{B}=\{x \in \mathbb{G}: 2 x=0\}$. After some simplification, the relations defining $\Delta_{n}$ may finally be presented as follows:

- $E_{m}^{2}=-E_{m}^{0}=H_{m}^{2}, E_{m}^{1}=H_{m}^{1}$.

- $T_{m}^{0}=T_{m}^{3}, T_{m}^{1}=T_{m}^{2}$.

- $H_{m}^{1}=H_{m-1}^{1} \in \mathbb{B}$.

- $Q_{m}^{2}=Q_{m-1}^{2} \in \mathbb{B}$.

- $H_{m}^{2}-H_{m-1}^{2}=T_{m}^{3}-T_{m}^{2}$.

- $Q_{m}^{4}-Q_{m}^{3}=T_{m}^{3}-T_{m-1}^{3}, Q_{m}^{3}-Q_{m}^{2}=T_{m}^{2}-T_{m-1}^{2}$.

\section{Order one invariants}

In this section we will show that the injection $u: V_{1} / V_{0} \rightarrow \Delta_{1}$ is surjective. Let us first give an explicit presentation of $\Delta_{1}$. We see from the presentation of the relations appearing in the end of the previous section, that a function $g \in \Delta_{1}$ may be assigned arbitrary values in $\mathbb{G}$ for the symbols $\left\{T_{m}^{a}\right\}_{a=2,3, m \in \mathbb{Z}}, H_{0}^{2}$ and arbitrary values in $\mathbb{B}$ for the two symbols $H_{0}^{1}, Q_{0}^{2}$. Once this is done then the value of $g$ on all other symbols is uniquely determined, namely: 
1. $H_{m}^{1}=H_{0}^{1}$ for all $m$.

2. $H_{m}^{2}=H_{0}^{2}+\sum_{k=1}^{m}\left(T_{k}^{3}-T_{k}^{2}\right)$ for $m \geq 0$.

3. $H_{m}^{2}=H_{0}^{2}-\sum_{k=m+1}^{0}\left(T_{k}^{3}-T_{k}^{2}\right)$ for $m<0$.

4. $E_{m}^{0}=-H_{m}^{2}, E_{m}^{1}=H_{m}^{1}, E_{m}^{2}=H_{m}^{2}$ for all $m$.

5. $T_{m}^{0}=T_{m}^{3}, T_{m}^{1}=T_{m}^{2}$ for all $m$.

6. $Q_{m}^{2}=Q_{0}^{2}$ for all $m$.

7. $Q_{m}^{3}=Q_{m}^{2}+T_{m}^{2}-T_{m-1}^{2}$ for all $m$.

8. $Q_{m}^{4}=Q_{m}^{3}+T_{m}^{3}-T_{m-1}^{3}$ for all $m$.

Let $X$ denote the set of symbols $\left\{T_{m}^{a}\right\}_{a=2,3, m \in \mathbb{Z}} \cup\left\{H_{0}^{2}\right\}$ then we have obtained that $\Delta_{1}(\mathbb{G}) \cong \mathbb{G}^{X} \oplus \mathbb{B} \oplus \mathbb{B}$ where $\mathbb{G}^{X}$ denotes the group of all functions from $X$ to $\mathbb{G}$. We can also present $\Delta_{1}(\mathbb{G})$ through a universal object as follows: We define a universal Abelian group $\mathbb{G}_{U}$ by the Abelian group presentation $\mathbb{G}_{U}=$ $\left\langle\left\{t_{m}^{a}\right\}_{a=2,3, m \in \mathbb{Z}}, h_{0}^{2}, h_{0}^{1}, q_{0}^{2} \mid 2 h_{0}^{1}=2 q_{0}^{2}=0\right\rangle$. Then we define the universal element $g_{U} \in \Delta_{1}\left(\mathbb{G}_{U}\right)$ by $g_{U}\left(T_{m}^{a}\right)=t_{m}^{a}(a=2,3), g_{U}\left(H_{0}^{2}\right)=h_{0}^{2}, g_{U}\left(H_{0}^{1}\right)=h_{0}^{1}$, $g_{U}\left(Q_{0}^{2}\right)=q_{0}^{2}$ and the value of $g_{U}$ on the other symbols of $\mathcal{C}_{1}$ is determined by formulae 1-8 above, so indeed $g_{U} \in \Delta_{1}\left(\mathbb{G}_{U}\right)$. Then for arbitrary Abelian group $\mathbb{G}$ we have $\Delta_{1}(\mathbb{G}) \cong \operatorname{Hom}\left(\mathbb{G}_{U}, \mathbb{G}\right)$ where the isomorphism maps a homomorphism $\phi \in \operatorname{Hom}\left(\mathbb{G}_{U}, \mathbb{G}\right)$ to the function $\phi \circ g_{U} \in \Delta_{1}(\mathbb{G})$. We will show that there is an order 1 invariant $f_{U}: I_{0} \rightarrow \mathbb{G}_{U}$ such that its extension to $I_{1}$ induces $g_{U}$ on $\mathcal{C}_{1}$, i.e. $u\left(f_{U}\right)=g_{U}$. It will follow that for any group $\mathbb{G}, u: V_{1} / V_{0} \rightarrow \Delta_{1}(\mathbb{G})$ is surjective, since if $g \in \Delta_{1}(\mathbb{G})$ and $g=\phi \circ g_{U}$ where $\phi \in \operatorname{Hom}\left(\mathbb{G}_{U}, \mathbb{G}\right)$ then $u\left(\phi \circ f_{U}\right)=g$.

We choose a base immersion $i_{0} \in I_{0}$ once and for all. Given any $i \in I_{0}$ we take a generic regular homotopy from $i_{0}$ to $i$ i.e. a path in $\mathcal{A}$ from $i_{0}$ to $i$ transverse with respect to the global stratification. The value of $f_{U}$ on $i$ will be the sum with signs, of the values of $g_{U}$ on the CEs that we pass along our path from $i_{0}$ to $i$, where the signs are determined by whether we are passing the given $\mathrm{CE}$ in the direction of its co-orientation. (Note that by definition of $\mathbb{G}_{U}$, whenever there is no co-orientation then the element we are adding is of order 2). We must show that this sum is independent of our choice of path, or equivalently that it is 0 along any closed path. First we observe that it is 0 on null-homotopic loops. Indeed, by slightly deforming the null-homotopy, we may assume it too is transverse with respect to the stratification. We may then break the null-homotopy into small pieces and clearly the value accumulated when going around our loop is the sum of the values going around each little piece. For appropriate partition there will be four types of such pieces according to their intersection with the strata, this intersection may

1. be empty,

2. be an arc of $I_{1}$,

3. be two intersecting arcs of $I_{1}$ (their intersection point being in $I_{2}$ ),

4. contain a point of local codim 2. 
In all four cases the sum going around the little piece is 0 . For cases 1 and 2 this is trivial. For case 3 this is true since local motion near one of the CEs does not effect the symbol $R_{m}^{a}$ attached to the other CE. For case 4 this is true since $g_{U} \in \Delta_{1}\left(\mathbb{G}_{U}\right)$.

Once we have established that this sum in $\mathbb{G}_{U}$ is 0 for any null-homotopic loop, we have a well defined homomorphism $\pi_{1}(\mathcal{A}) \rightarrow \mathbb{G}_{U}$ and we must show that this homomorphism is 0 . We first show this for the case $F=S^{2}$ in which case there is only one regular homotopy class, which we still name $\mathcal{A}$. Now $\pi_{1}(\mathcal{A})=\mathbb{Z} \oplus \mathbb{Z} / 2$ where the unique order 2 element is represented by one full rotation of $S^{2}$ in $\mathbb{R}^{3}$. Such rotation passes through no CEs at all, and so gives 0 as needed. Let $K \subseteq \pi_{1}(\mathcal{A})$ be the subgroup generated by this order 2 element in $\pi_{1}(\mathcal{A})$ and let $P=P\left(S^{2}\right)=\pi_{1}(\mathcal{A}) / K$, then we are left with showing that the map induced on $P(\cong \mathbb{Z})$ is 0 .

If $H_{t}: S^{2} \rightarrow \mathbb{R}^{3}$ is a regular homotopy, then attaching to $H_{t}$ one or more of the superscripts $\mathbb{R}^{3}, S^{2}$ and $T$ will denote respectively, composition from the left with the antipodal map of $\mathbb{R}^{3}$, composition from the right with the antipodal map of $S^{2}$ and reversal of time. Note that these three operations on $H_{t}$ commute with each other. Let $p: S^{2} \rightarrow \mathbb{R} P^{2}$ be the double covering and let $i: \mathbb{R} P^{2} \rightarrow \mathbb{R}^{3}$ be some immersion. Let $s=i \circ p$ then $s: S^{2} \rightarrow \mathbb{R}^{3}$ is an immersion satisfying $s(-x)=s(x)$. Let $H_{t}$ be a regular homotopy from the inclusion $e: S^{2} \rightarrow \mathbb{R}^{3}$ to $s$. Let $G_{t}=H_{t} *\left(H_{t}^{S^{2}, T}\right)$ where $*$ denotes concatenation from left to right, then $G_{t}$ is a regular homotopy from $e$ to $-e$ i.e. an eversion of the sphere. Also $G_{t}^{S^{2}, T}=G_{t}$. We now define $F_{t}=G_{t} * G_{t}^{\mathbb{R}^{3}}=G_{t} *\left(G_{t}^{S^{2}, T}\right)^{\mathbb{R}^{3}}=G_{t} *\left(G_{t}^{S^{2}, \mathbb{R}^{3}}\right)^{T}$. From the presentation of $F_{t}$ as $G_{t} * G_{t}^{\mathbb{R}^{3}}$ we can see that there are isotopies $U_{t}: \mathbb{R}^{3} \rightarrow \mathbb{R}^{3}$ and $V_{t}: S^{2} \rightarrow S^{2}$ such that $U_{t} \circ F_{t} \circ V_{t}$ satisfies the conditions of [N1] Proposition 2.1. It follows that $F_{t}$ represents an odd power of the generator of $P$. We also have $F_{t}=G_{t} *\left(G_{t}^{S^{2}, \mathbb{R}^{3}}\right)^{T}$ and let $G_{t}^{\prime}$ be a generic regular homotopy which is a slight perturbation of $G_{t}\left(G_{t}\right.$ was highly non-generic $)$, then $F_{t}^{\prime}=G_{t}^{\prime} *\left(G_{t}^{\prime S^{2}, \mathbb{R}^{3}}\right)^{T}$ will still represent an odd power of the generator of $P$ (though it will not be precisely equal to $G_{t}^{\prime} * G_{t}^{\prime \mathbb{R}^{3}}$ ). We now observe that reversing the orientations of both $S^{2}$ and $\mathbb{R}^{3}$ simultaneously, preserves all our definitions of types, degrees and co-orientations of CEs and so $G_{t}^{\prime S^{2}, \mathbb{R}^{3}}$ produces precisely the same element in $\mathbb{G}_{U}$ as $G_{t}^{\prime}$ and so $\left(G_{t}^{\prime S^{2}, \mathbb{R}^{3}}\right)^{T}$ produces the negative of that element. So we get that the value on the loop $F_{t}^{\prime}$ is 0 . Since $F_{t}^{\prime}$ represents an odd power of the generator of $P$ and since in $\mathbb{G}_{U}$ an odd multiple of any non-zero element is still non-zero, we get that the value is 0 on the generator of $P$ and so on all $P$. This completes the proof for the case $F=S^{2}$.

Now let $F$ be of higher genus and let $\mathcal{A}$ be a given regular homotopy class. Again $\pi_{1}(\mathcal{A}) \cong \mathbb{Z} \oplus \mathbb{Z} / 2$. Again our claim is clear for $K \subseteq \pi_{1}(\mathcal{A})$ which is defined as above and so again it is enough to look at the map induced on $P=$ $P(F, \mathcal{A})=\pi_{1}(\mathcal{A}) / K$. 
Looking back for a moment at $S^{2}$ let $H_{t}$ be a loop representing a generator of $P\left(S^{2}\right)$ and we may assume $H_{t}$ fixes a given disc $D \subseteq S^{2}$. Choose a point $x$ on a ray perpendicular to the fixed image of $D$ in $\mathbb{R}^{3}, x$ being far enough so that the image of $H_{t}$ never passes $x$. Now change the constant embedding of $D$ to be one with a thin "thorn" pulled out of $D$ and embedded along this ray, reaching $x$. The new loop $H_{t}^{\prime}$ obtained in this way also represents the generator of $P\left(S^{2}\right)$ and has the property that a given point in the fixed disc $D$ (namely, the tip of the thorn) does not participate in any self intersections throughout $H_{t}^{\prime}$. Since we have already proved our result for $S^{2}$, we know that the symbols encountered along the loop $H_{t}^{\prime}$ add up to 0 in the group $\mathbb{G}_{U}$. Now take a tiny immersion of $F$ in $I_{0}$, located near the tip of our fixed thorn, and connect sum it with $H_{t}^{\prime}$ (for all $t$ ), obtaining a loop $F_{t}: F \rightarrow \mathbb{R}^{3}$ in $\mathcal{A}$. It follows from the proof of [N1] Theorem 3.4 that this loop $F_{t}: F \rightarrow \mathbb{R}^{3}$ represents a generator of $P(F, \mathcal{A})$. Since the tip of the thorn was far away from any self intersections occurring in $H_{t}^{\prime}$, the tiny fixed immersion of $F$ will not cause any new CEs and will not affect the degrees of the CEs of $H_{t}^{\prime}$ and so will not alter the sum of symbols attained by $H_{t}^{\prime}$, which we know is 0 in $\mathbb{G}_{U}$. We conclude that $f_{U}$ is well defined; it is evident that $f_{U}$ is of order 1 and $u\left(f_{U}\right)=g_{U}$. This completes the proof of our main result:

Theorem 5.1. For any closed orientable surface $F$, regular homotopy class $\mathcal{A}$ of immersions of $F$ into $\mathbb{R}^{3}$ and Abelian group $\mathbb{G}$, the injection $u: V_{1} / V_{0} \rightarrow \Delta_{1}$ is surjective.

Remark 5.2. If $i \in I_{n-1}$ and $x \in i(F)$ is not a self intersection point then let $B$ be a small neighborhood of $x$ in $\mathbb{R}^{3}$ and $D=i^{-1}(B) \subseteq F$. By grafting a generator of $P\left(S^{2}\right)$ as above, we may move $D$ in $B$ as to produce a loop in $I_{n-1} \cup I_{n} \subseteq \mathcal{A}$ which generates $P(F, \mathcal{A})$. A priori such a loop could have added another relation to those with which we have defined $\Delta_{n}$, but it follows from the work of this section that such loop does not add any new relation. (This is in contrast with knot theory where the analogous loop produces the 1-term relation. Recall that we are viewing knots in the space of immersions rather than in the space of all maps).

\section{Eversions of the sphere}

We present an application of Theorem 5.1. Let $e: S^{2} \rightarrow \mathbb{R}^{3}$ denote the inclusion. An eversion of the sphere is a regular homotopy $H_{t}: S^{2} \rightarrow \mathbb{R}^{3}$ between $e$ and $-e$. In [MB] it has first been established that the number of CEs of type $Q$ occurring in any generic eversion of the sphere, is odd.

Given a generic eversion of the sphere, and given a class $v$ of CEs, we denote by $N(v)$ the number of CEs of type $v$ occurring during this eversion, counted with signs if all CEs of $v$ have a co-orientation, and counted $\bmod 2$ if $v$ includes CEs of type $H^{1}$ or $Q^{2}$. We may thus write the result of [MB] as follows: For any eversion of the sphere, 
(1) $N(Q)=1 \bmod 2$.

In $[G]$, three more such equations satisfied by any eversion of the sphere have been established, namely,

(2) $N(T)=0$

(3) $N\left(H^{2}\right)+N\left(E^{2}\right)-N\left(E^{0}\right)=-1$

(4) $N\left(H^{1}\right)+N\left(E^{1}\right)=0 \bmod 2$.

We now show how to deduce these four equations from our work, and then establish an infinite list of new such equations satisfied by any eversion of the sphere.

The Morin eversion described in [M] has 14 CEs. By direct inspection they are: (a \pm sign appears where there is no co-orientation)

$$
E_{1}^{0}+E_{2}^{2}+T_{2}^{2} \pm H_{2}^{1} \pm H_{2}^{1}+T_{2}^{1}-Q_{2}^{4}-H_{1}^{2}-T_{1}^{2} \pm H_{0}^{1} \pm H_{0}^{1}-T_{1}^{1}-E_{0}^{0}-E_{1}^{2} .
$$

Via the relations defining $\Delta_{1}$ this simplifies to:

$$
Q_{0}^{2}-H_{1}^{2}
$$

And so any order one invariant $f$ on $\operatorname{Imm}\left(S^{2}, \mathbb{R}^{3}\right)$ based at $e$, (i.e. having $f(e)=$ 0) will satisfy:

$$
f(-e)=u(f)\left(Q_{0}^{2}\right)-u(f)\left(H_{1}^{2}\right) .
$$

Recall that we have denoted $X=\left\{T_{m}^{a}\right\}_{a=2,3, m \in \mathbb{Z}} \cup\left\{H_{0}^{2}\right\}$ and let us now denote $Y=X \cup\left\{H_{0}^{1}, Q_{0}^{2}\right\}$. By Theorem 5.1 and the opening paragraph of Section 5, an order one invariant $f$ on $\operatorname{Imm}\left(S^{2}, \mathbb{R}^{3}\right)$, with values in an Abelian group $\mathbb{G}$ and based at $e$, may be defined by choosing arbitrary values for $u(f)(x)$ in $\mathbb{G}$ for all symbols $x \in X$ and arbitrary values in $\mathbb{B}=\{a \in \mathbb{G}: 2 a=0\}$ for $H_{0}^{1}$ and $Q_{0}^{2}$. Then the values on $\mathcal{C}_{1}-Y$ are determined by formulae $1-8$ of Section 5 .

Let $f_{1}$ be the $\mathbb{Z} / 2$ valued invariant with $g_{1}=u\left(f_{1}\right)$ satisfying $g_{1}\left(Q_{0}^{2}\right)=1 \in$ $\mathbb{Z} / 2$ and $g_{1}$ is 0 on all other symbols of $Y$. By $1-8$ of Section 5 we then get $g_{1}\left(Q_{m}^{a}\right)=1 \in \mathbb{Z} / 2$ for any $a, m$ and $g_{1}$ is 0 on all other symbols of $\mathcal{C}_{1}$. So for any eversion of the sphere, $N(Q)=f_{1}(-e)=g_{1}\left(Q_{0}^{2}\right)-g_{1}\left(H_{1}^{2}\right)=1 \bmod 2$ which is (1) above. Let $f_{2}$ be the $\mathbb{Z}$ valued invariant defined by $g_{2}\left(T_{m}^{a}\right)=1$ for $a=2,3$ and all $m$ and $g_{2}\left(H_{0}^{2}\right)=g_{2}\left(H_{0}^{1}\right)=g_{2}\left(Q_{0}^{2}\right)=0$. By $1-8$ of Section $5, g_{2}\left(T_{m}^{a}\right)=1$ for all $a, m$ and $g_{2}$ is 0 on all other symbols in $\mathcal{C}_{1}$ and so for any eversion of the sphere $N(T)=f_{2}(-e)=g_{2}\left(Q_{0}^{2}\right)-g_{2}\left(H_{1}^{2}\right)=0$ which is (2) above. In the same way define the $\mathbb{Z}$ valued invariant $f_{3}$ by $g_{3}\left(H_{0}^{2}\right)=1$ and $g_{3}$ is 0 on the rest of $Y$. This leads to $g_{3}\left(H_{m}^{2}\right)=g_{3}\left(E_{m}^{2}\right)=1, g_{3}\left(E_{m}^{0}\right)=-1$ for all $m$ and $g_{3}$ is 0 on the rest of $\mathcal{C}_{1}$, getting for any eversion of the sphere $N\left(H^{2}\right)+N\left(E^{2}\right)-N\left(E^{0}\right)=f_{3}(-e)=g_{3}\left(Q_{0}^{2}\right)-g_{3}\left(H_{1}^{2}\right)=-1$ which is (3). Finally Let $g_{4}$ be the $\mathbb{Z} / 2$ valued invariant defined by $g_{4}\left(H_{0}^{1}\right)=1 \in \mathbb{Z} / 2$ and $g_{4}$ is 0 on the rest of $Y$. This leads to $g_{4}\left(H_{m}^{1}\right)=g_{4}\left(E_{m}^{1}\right)=1 \in \mathbb{Z} / 2$ for all $m$ and $g_{4}$ is 0 on the rest of $\mathcal{C}_{1}$, getting for any eversion of the sphere $N\left(H^{1}\right)+N\left(E^{1}\right)=f_{4}(-e)=g_{4}\left(Q_{0}^{2}\right)-g_{4}\left(H_{1}^{2}\right)=0 \bmod 2$ which is (4). 
In the same way, we now produce an infinite list of new, finer equations, satisfied by any eversion of the sphere. For any pair $b, r$ with $b \in\{2,3\}, r \in \mathbb{Z}$ we define a $\mathbb{Z}$ valued invariant $f_{b r}$ by $g_{b r}\left(T_{r}^{b}\right)=1$ and if $r \leq 0$ then also $g_{b r}\left(H_{0}^{2}\right)=(-1)^{b-1}$, and $g_{b r}$ is 0 on the rest of $Y$. This leads, by $1-8$ of Section 5 to the following: $g_{b r}\left(T_{r}^{3-b}\right)=g_{b r}\left(T_{r}^{b}\right)=1, g_{b r}\left(H_{m}^{2}\right)=g_{b r}\left(E_{m}^{2}\right)=$ $-g_{b r}\left(E_{m}^{0}\right)=(-1)^{b-1}$ for $m \geq r, g_{b r}\left(Q_{r}^{a}\right)=1$ for $a \geq b+1, g_{b r}\left(Q_{r+1}^{a}\right)=-1$ for $a \geq b+1$, and $g_{b r}$ is 0 on the rest of $\mathcal{C}_{1}$. This gives for any eversion of the sphere $N\left(T_{r}^{b}\right)+N\left(T_{r}^{3-b}\right)+(-1)^{b-1}\left(N\left(H_{\geq r}^{2}\right)+N\left(E_{\geq r}^{2}\right)-N\left(E_{\geq r}^{0}\right)\right)+N\left(Q_{r}^{\geq b+1}\right)-$ $N\left(Q_{r+1}^{\geq b+1}\right)=f_{b r}(-e)=g_{b r}\left(Q_{0}^{2}\right)-g_{b r}\left(H_{1}^{2}\right)$, where e.g. $N\left(H_{\geq r}^{2}\right)$ means the number (with signs) of CEs occurring during the eversion which are of type $H_{m}^{2}$ with $m \geq r$. So finally:

Proposition 6.1. For each pair $b, r$ with $b \in\{2,3\}, r \in \mathbb{Z}$, the following equation is satisfied by any eversion of the sphere:

$$
\begin{gathered}
N\left(T_{r}^{b, 3-b}\right)+(-1)^{b-1}\left(N\left(H_{\geq r}^{2}\right)+N\left(E_{\geq r}^{2}\right)-N\left(E_{\geq r}^{0}\right)\right) \\
\quad+N\left(Q_{r}^{\geq b+1}\right)-N\left(Q_{r+1}^{\geq b+1}\right)= \begin{cases}(-1)^{b} & r \leq 1 \\
0 & r>1\end{cases}
\end{gathered}
$$

We note that equations (2) and (3) above follow from Proposition 6.1. Indeed, given an eversion of the sphere $H_{t}: S^{2} \rightarrow \mathbb{R}^{3}$, let $k$ and $K$ be the minimal and maximal degrees occurring in $H_{t}$ respectively. For $r=\min (1, k-2)$ (and either b) Proposition 6.1 yields $N\left(H^{2}\right)+N\left(E^{2}\right)-N\left(E^{0}\right)=-1$ for $H_{t}$. Summing the equation of Proposition 6.1 over $k-1 \leq r \leq K$ and $b=2,3$ yields $N(T)=0$ for $H_{t}$. (In fact $N(T)=0$ is an obvious equation following from the fact that the number of triple points in both $e$ and $-e$ is 0 ).

\section{Higher order invariants}

We now show that for $\mathbb{G}=\mathbb{Z} / 2$ and $n>1$ the map $u: V_{n} / V_{n-1} \rightarrow \Delta_{n}$ is not surjective. We define a function $g: \mathcal{C}_{n} \rightarrow \mathbb{Z} / 2$ as follows: If $x \in \mathcal{C}_{n}$ includes at least one symbol which is not of type $Q$, then $g(x)=0$ and if all symbols in $x$ are of type $Q$ then $g(x)=1$. One verifies directly that $g$ satisfies the relations appearing in the end of Section 4 and so $g \in \Delta_{n}\left(\mathbb{Z}_{2}\right)$. We will show that there is no order $n$ invariant $f$ such that $u(f)=g$. Let $f: I_{0} \rightarrow \mathbb{Z} / 2$ be an invariant of order $n$ and assume $u(f)=g$. Since $\mathbb{G}=\mathbb{Z} / 2$, there is no need for co-orientations for the extension of $f$ and so $f$ extends to $I_{k}$ for any $k>0$, in particular to $I_{n-1}$. Let $i, j \in I_{n-1}$ be two AB equivalent immersions such that all $n-1$ CEs of $i$ and $j$ are of type $Q$. If $H_{t}$ is a (generic) AB equivalence between $i$ and $j$ then there are some finitely many times along $H_{t}$ for which an $n$th CE appears. By definition of 
$g$, the number mod 2 of such $n$th CEs which are of type $Q$ is $f(i)-f(j) \in \mathbb{Z} / 2$. In particular if $i=j$ then the number of such occurrences of an $n$th quadruple point is $0 \bmod 2$.

Now take some $i \in I_{n-1}$ with $n-1$ CEs located at $p_{1}, \ldots, p_{n-1}$ all of type $Q$ and furthermore the CE at $p_{1}$ is of type $Q^{2}$. Let $H_{t}$ be an $\mathrm{AB}$ equivalence from $i$ to itself which fixes $F$ in neighborhoods of $p_{2}, \ldots, p_{n-1}$ and reverses the co-orientation of the CE at $p_{1}$ (as in the proof of Proposition 3.5). Still assuming the existence of an $f$ such that $u(f)=g$, by the above discussion the number of quadruple point occurrences along $H_{t}$ is $0 \bmod 2$. Let $i^{\prime} \in I_{0}$ be an immersion which is obtained from $i$ by slightly deforming $i$ in a neighborhood of each $p_{k}, k=1, \ldots, n-1$. $H_{t}$ induces a regular homotopy $H_{t}^{\prime}$ from $i^{\prime}$ to an immersion which differs from $i^{\prime}$ only in a neighborhood of $p_{1}$ and there are $0 \bmod 2$ quadruple point occurrences along $H_{t}^{\prime}$, precisely those occurring during $H_{t}$. We complete $H_{t}^{\prime}$ to a closed loop ending at $i^{\prime}$ causing one more quadruple point to occur, namely that at $p_{1}$. And so we have constructed a loop in $I_{0} \cup I_{1}$ (transverse to $I_{1}$ ) with $1 \bmod 2$ quadruple point occurrences. This would imply (by our above discussion again) that the analogous order 1 invariant does not exist. But we have shown (Theorem 5.1) that for order 1, all invariants defined in this way do exist, a contradiction. (This order 1 invariant appeared as $f_{1}$ in Section 6.) We conclude that there is no order $n$ invariant $f$ such that $u(f)=g$ and so for $\mathbb{G}=\mathbb{Z} / 2$ and $n>1$ the map $u: V_{n} / V_{n-1} \rightarrow \Delta_{n}(\mathbb{Z} / 2)$ is not surjective.

We remark that the order 1 invariant whose existence has been used in the above proof, namely that order 1 invariant which counts the number mod 2 of quadruple points occurring along generic regular homotopies, has been studies in [N1], [N3]. In [N1] its existence has been established for all closed surfaces (not only orientable) and also the existence of the analogous invariant in general 3-manifolds, under certain conditions. In [N3] an explicit formula has been given for the value of this invariant on embeddings.

Another remark: In [N2] the notion of a $q$-invariant has been defined. This is an invariant $f: I_{0} \rightarrow \mathbb{Z} / 2$ such that its extension to $I_{1}$ satisfies that $f(i)=0$ if the CE of $i$ is not a quadruple point. It follows that $f(i)=0$ for any $i \in I_{k}$ $(k \geq 1)$ which includes at least one CE which is not a quadruple point. If $f$ is of order $n$ then this means that $u(f)(x)=0$ for any $x \in \mathcal{C}_{n}$ which includes at least one symbol which is not of type $Q$. It has been shown in [N2] and also follows from the relations defining $\Delta_{n}$ (end of Section 4), that if such $f$ of order $n$ exists, then $u(f)(x)=1$ for any $x \in \mathcal{C}_{n}$ for which all symbols are of type $Q$. So what we have shown in this section is that $q$-invariants of order $n>1$ do not exist, by this completing the work of [N2]. (The unique $q$-invariant of order 1 is the invariant discussed in the previous paragraph).

We conclude by showing that for any $\mathbb{G}$ there exist invariants of any order $n$. Let $p_{n}(x)=\left(\begin{array}{c}x+n \\ n\end{array}\right)$, then $p_{n}(x)$ is a polynomial of degree $n$ satisfying the relation: $p_{n}(x+1)-p_{n}(x)=p_{n-1}(x+1)$. Now fix an $n \geq 0$ and an element $0 \neq g \in \mathbb{G}$ 
and define $f: I_{0} \rightarrow \mathbb{G}$ by $f(i)=p_{n}(t(i)) g$ where $t(i)$ denotes half the number of triple points occurring in $i$. (The number of triple points in a stable immersion of a closed orientable surface into $\mathbb{R}^{3}$ is known to be even, see [B]). One shows inductively that for $i \in I_{k}, f(i)=0$ if at least one of the $k$ CEs of $i$ is not of type $T$ and $f(i)=p_{n-k}(t(i)+k) g$ if all $k$ CEs are of type $T$, where $t(i)$ is half the number of stable triple points occurring in $i$ (i.e. we do not count the type $T$ CEs as triple points) and where we use the permanent co-orientation of these type $T$ CEs when computing $f(i)$. In particular $f(i)=g \neq 0$ for any $i \in I_{n}$ for which all $n$ CEs are of type $T$ and $f(i)=0$ for all $i \in I_{n+1}$ and so $f$ is an invariant of order precisely $n$.

\section{References}

[B] Banchoff, T.F.: Triple points and surgery of immersed surfaces. Proc. Am. Math. Soc. 46, 407-413 (1974)

[G] Goryunov, V.V.: Local Invariants of Mappings of Surfaces into Three-Space. ArnoldGelfand Mathematical Seminars, Geometry and Singularity Theory. Birkhauser Boston Inc., 1997, pp. 223-255

[H] Hirsch, M.W.: Immersions of manifolds. Trans. Am. Math. Soc. 93, 242-276 (1959)

[HK] Hobbs, C.A., Kirk, N.P.: On the classification and bifurcation of multigerms of maps from surfaces to 3-space. Mathematica Scandinavica 89, 57-96 (2001)

[K] Kontsevich, M.: Vassiliev's knot invariants. I.M. Gelfand Seminar, Advances in Soviet Mathematics 16, Part 2, Am. Math. Soc., Providence, RI, 1993, pp. 137-150

[M] Max, N.: Turning a sphere inside out, a guide to the film. Computers in Mathematics, Marcel Dekker Inc., 1990, pp. 334-345

[MB] Max, N., Banchoff, T.: Every Sphere Eversion Has a Quadruple Point. Contributions to Analysis and Geometry, John Hopkins University Press, 1981, pp. 191-209

[N1] Nowik, T.: Quadruple Points of Regular Homotopies of Surfaces in 3-Manifolds. Topology 39, 1069-1088 (2000)

[N2] Nowik, T.: Finite order $q$-invariants of immersions of surfaces into 3-space. Mathematische Zeitschrift 236, 215-221 (2001)

[N3] Nowik, T.: Automorphisms and embeddings of surfaces and quadruple points of regular homotopies. J. Diff. Geom. 58, 421-455 (2001)

[S] Smale, S.: A classification of immersions of the two-sphere. Trans. Am. Math. Soc. 90, 281-290 (1958) 